Arch. Tierz., Dummerstorf 47 (2003) 4, 351-358

Aus dem Institut für Tierzucht und Vererbungsforschung der Tierärztlichen Hochschule Hannover

FRERICH DE VRIES, HENNING HAMANN und OTTMAR DISTL

\title{
Schätzung genetischer Parameter für Landschafrassen
}

\begin{abstract}
Title of the paper: Estimation of genetic parameters in land sheep breeds

The objective of the present study was a genetic statistical analysis of performance traits recorded at the day of licensing in land sheep breeds. The performance traits score for muscle mass, type and wool quality were analysed for the breeds German Polled Heath, German Grey Heath, Bentheim, German White Heath and Coburg from breeding regions in Lower Saxony and Westphalia. Systematic fixed effects of herd-year-season, test day, sex, birth rearing type and the linear covariate age at licensing were included in the statistical models to estimate the variance and covariance components. There were high additive genetic correlations between muscle mass and type. The estimates of additive genetic correlations between wool quality and type or wool quality and muscle mass were moderate. The heritabilities estimated separately for each breed ranged between $\mathrm{h}^{2}=0.06$ and $\mathrm{h}^{2}=0.16$ for muscle mass and between $\mathrm{h}^{2}=0.04$ and $\mathrm{h}^{2}=0.09$ for type. The biggest range of heritabilities was estimated for wool quality with $\mathrm{h}^{2}=0.03$ to $\mathrm{h}^{2}=0.14$.
\end{abstract}

Key Words: land sheep breeds, genetic parameters, muscle mass, type traits, wool quality

\section{Zusammenfassung}

Das Ziel der Studie war die genetisch-statistische Analyse von bei der Körung und Herdbuchaufnahme registrierten Merkmalen von Landschafrassen, deren Bedeutung in den letzten Jahren sehr zugenommen hat. Untersucht wurden die Rassen Weiße hornlose Heidschnucke, Graue gehörnte Heidschnucke, Bentheimer Landschaf, Weiße gehörnte Heidschnucke und Coburger Fuchsschaf aus dem niedersächsischen und westfälischen Zuchtgebiet. Als Merkmale standen die Bonitierung der Bemuskelung, der Wolle sowie des Exterieurs zur Verfügung. Die fixen Effekte Herde-Jahr-Saison, Messtag, Geschlecht, Geburtstyp sowie die lineare Kovariable Alter beim Messtag wurden als systematische Effekte zur Ermittlung der Varianz- und Kovarianzkomponenten berücksichtigt. Zwischen den Merkmalen Bemuskelung und Exterieur wurde eine hohe additiv-genetische Korrelation geschätzt. Dagegen wurde nur eine mittlere additiv-genetische Korrelation zwischen den Merkmalen Wolle und Bemuskelung sowie Wolle und Exterieur geschätzt. Die Heritabilitäten der einzelnen Rassen für das Merkmal Bemuskelung lagen zwischen $\mathrm{h}^{2}=0,06$ und $\mathrm{h}^{2}=0,16$ sowie für das Merkmal Exterieur zwischen $\mathrm{h}^{2}=0,04$ und $h^{2}=0,09$. Für das Merkmal Wolle bestand die größte Spannweite in den Heritabilitätsschätzwerten mit $h^{2}=0,03$ bis $h^{2}=0,14$.

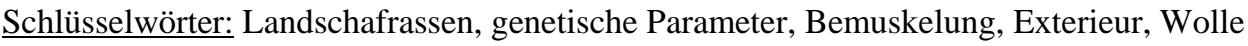

1. $\quad$ Einleitung

Die Zahl der in das Zuchtbuch eingetragenen Landschafe nimmt kontinuierlich zu. Dies hängt nicht nur mit dem zunehmenden Beliebtheitsgrad dieser Schafrassen zusammen, sondern auch mit der wieder zunehmenden Bedeutung der Schafe in der Landschaftspflege. Schonende Trittwirkung, breites Futteraufnahmespektrum und stark selektierendes Fressverhalten verleihen den Landschafrassen die besondere Eignung für den Einsatz in der Landschaftspflege. Insbesondere alte, bodenständige Rassen, wie schon am Name der Rasse zu erkennen (Bentheimer Landschaf, Coburger Fuchsschaf), weisen eine besondere Eignung und Verbundenheit mit bestimmten Regionen und Lebensräumen auf. Viele der Landschafrassen gehörten in den letzten Jah- 
ren zu den bedrohten Rassen. Nachdem sich die Populationen einiger Landschafrassen nun zahlenmäßig einigermaßen erholt haben, kann der Selektion auf ökologisch und wirtschaftlich wichtige Merkmale größere Bedeutung geschenkt werden. Auf der Grundlage der für die Zuchtauswahl im Rahmen der Körung und Herdbuchaufnahme verwendeten Daten sollen genetische Parameter geschätzt werden. Die hierbei untersuchten drei Merkmale stellen inzwischen die Hauptmerkmale bei der Selektion der Zuchttiere dar, was die Analyse der genetischen Parameter dieser Merkmale erforderlich macht.

\section{2.}

\section{Material und Methode}

Für die Untersuchung der genetischen Parameter standen Herdbuchdaten aus den Jahren 1992 bis 2002 der Landesschafzuchtverbände aus Niedersachsen und Westfalen zur Verfügung. Zur Analyse wurden die Landschafrassen Graue gehörnte Heidschnucke (GgH), Weiße gehörnte Heidschnucke (WgH), Weiße hornlose Heidschnucke (WhH), Bentheimer Landschaf (Bent), und Coburger Fuchsschaf (CoF) ausgewählt. Insgesamt standen so für 12474 Landschafe Daten zur Verfügung (Tab. 1). Die zu analysierenden Merkmale wurden bei Bocklämmern am Tage der Körung und von weiblichen Tieren am Tag der Zuchtbuchaufnahme erhoben. Untersucht wurden die Bonitierung der Bemuskelung, der Wollqualität und des Exterieurs. Diese Merkmale wurden mit Noten von 1 bis 9 bewertet, wobei die Note 9 die beste Bewertung darstellte. Da alle Merkmale zusammen erhoben wurden, lagen gleich viele Eintragungen dieser drei Merkmale vor. Aufgrund unterschiedlicher Organisation der Zuchtbuchaufnahme bzw. Körung in den einzelnen Verbänden resultierte eine große Anzahl unterschiedlich großer Vergleichsgruppen, bei denen an demselben Erfassungstag die Daten erhoben wurden. Für diese Analyse wurden die Messtage deshalb in Messtagsklassen eingeteilt, die mindestens sechs Beobachtungen enthielten. Pro Rasse variierte die Anzahl dieser Messtagsklassen von 26 (WgH) bis 54 (Bent). Die Geburtssaison wurde in eine Haupt- (Januar bis März) und eine Nebenablammsaison (April bis Dezember) unterteilt und zu Herde-Jahr-Saisonklassen (HJS) zusammengefasst. HerdeJahr-Saisonklassen mit nur einer Beobachtung wurden nicht berücksichtigt. Die Anzahl der Herde-Jahr-Saisonklassen variierte pro Rasse zwischen 79 (CoF, WgH) und 287 (Bent). Der Geburtstyp eines Tieres gibt an, ob das Tier als Einling, Zwilling oder Drilling geboren wurde. Bei den Landschafen wird die Geburt nur eines Lammes angestrebt, es treten kaum Zwillings- und Drillingsgeburten auf. Diese wurden deshalb in einer Klasse zusammengefasst. Die Zahl der im Pedigree vertretenen Tiere wird in Tabelle 6 angegeben.

Zur genetisch-statistischen Analyse dieser drei Leistungsmerkmale wurde ein Modell mit Hilfe der Prozedur GLM (SAS, Version 8.2, 2003) entwickelt, das die in der Literatur genannten Einflussfaktoren enthielt. Hierbei wurden die Einflussfaktoren auf Signifikanz getestet und die Werte des Bestimmtheitsmaßes in unterschiedlichen Modellen miteinander verglichen. Die Auswertung erfolgte hierbei für jede Rasse getrennt. Im Modell wurden Herde-Geburtsjahr-Geburtssaison, Messtag, Geschlecht und Geburtstyp als fixe Effekte, das Alter als lineare Kovariable sowie der additiv-genetische Effekt des Tieres und der Restfehler als zufällige Effekte berücksichtigt. Der additiv-genetische Effekt des Tieres bezieht sich auf das Tier selber, seine verwandten Tiere mit und ohne Beobachtungen. Für die Schätzung genetischer Parameter wurde das nachfolgende lineare Tiermodell verwendet: 
Modell:

\begin{tabular}{|c|c|}
\hline$Y_{\mathrm{ijklm}}=$ & $\mu+$ HJS $_{i}+$ MTKL $_{j}+G_{k}+G_{l}+b$ Alt $_{i j k l m}+a_{i j k l m}+e_{i j k l m}$ \\
\hline$Y_{\mathrm{ijklm}}$ & $\begin{array}{l}\text { : Beobachtungwert für das jeweilige Leistungsmerkmal des ijklm-ten } \\
\text { Tieres }\end{array}$ \\
\hline$\mu$ & : Modellkonstante \\
\hline $\mathrm{HJS}_{\mathrm{i}}$ & $\begin{array}{l}\text { : fixer Effekt der Herde-Geburtsjahr-Geburtssaison (i = 79, 79, 253, 271, } \\
287 \text { für CoF, WgH, WhH, GgH, Bent) }\end{array}$ \\
\hline MTKL $_{\mathrm{j}}$ & $\begin{array}{l}\text { : fixer Effekt der Messtagsklasse }(\mathrm{j}=26,35,45,52,54 \text { für WgH, CoF, } \\
\text { GgH, WhH, Bent) }\end{array}$ \\
\hline $\mathrm{G}_{\mathrm{k}}$ & : fixer Effekt des Geschlechts $(\mathrm{k}=1-2)$ \\
\hline $\mathrm{GT}_{1}$ & : fixer Effekt des Geburtstyps (l = 1 - 2) \\
\hline $\mathrm{b}$ & : linearer Regressionskoeffizient \\
\hline $\mathrm{Alt}_{\mathrm{ijklm}}$ & : Alter des Tieres bei der Körung/Zuchtbuchaufnahme \\
\hline$a_{i j k l m}$ & $\begin{array}{l}\text { : zufälliger additiv-genetischer Tiereffekt }(\mathrm{n}=1-1338,1692,5251 \text {, } \\
\text { 6714, } 7511 \text { für WgH, CoF, Bent, GgH, WhH) }\end{array}$ \\
\hline$e_{i j k l m}$ & : zufälliger Resteffekt \\
\hline
\end{tabular}

Mit dem Programm VCE4, Version 4.2.5, (GROENEVELD, 1998) wurden unter Berücksichtigung der signifikanten systematischen Effekte die Varianzkomponenten sowie die additiv-genetischen Korrelationen für jede Rasse mittels Residual Maximum Likelihood (REML) geschätzt. Die additiv-genetischen Korrelationen wurden für alle Landschafrassen in einem Modell geschätzt, das den fixen Effekt Rasse enthielt.

Tabelle 1

Anzahl der Beobachtungen (n), Mittelwerte $(\bar{X})$ und Standardabweichungen der Merkmale Bemuskelung, Exterieur und Wolle sowie des Alters in Tagen bei der Körung/Zuchtbuchaufnahme für Landschafrassen (Number of records (n), means $(\bar{X})$ and standard devations (s) of the traits muscle mass, type and wool quality as well as age in days at licensing of land sheep breeds)

\begin{tabular}{|c|c|c|c|c|c|c|c|c|c|c|c|c|}
\hline \multirow{2}{*}{ Rasse } & \multirow{2}{*}{$\begin{array}{c}\text { Anzahl } \\
\mathrm{n}\end{array}$} & \multicolumn{3}{|c|}{ Bemuskelung } & \multicolumn{3}{|c|}{ Exterieur } & \multicolumn{2}{|c|}{ Wolle } & \multicolumn{3}{|c|}{ Alter } \\
\hline & & $\bar{x}$ & \pm & $\mathrm{s}$ & $\bar{x}$ & \pm & $\mathrm{s}$ & $\bar{x}$ & $\pm \quad s$ & $\bar{x}$ & \pm & $\mathrm{s}$ \\
\hline $\begin{array}{l}\text { Weiße hornlose } \\
\text { Heidschnucke }\end{array}$ & 4041 & 7,18 & & 0,74 & 7,08 & \pm & 0,72 & 7,16 & $\pm 0,66$ & 351 & \pm & 130 \\
\hline $\begin{array}{l}\text { Graue gehörnte } \\
\text { Heidschnucke }\end{array}$ & 3799 & 7,00 & \pm & 0,80 & 6,81 & \pm & 0,78 & 6,98 & $\pm 0,71$ & 468 & \pm & 101 \\
\hline $\begin{array}{l}\text { Bentheimer } \\
\text { Landschaf }\end{array}$ & 3170 & 7,06 & \pm & 0,63 & 6,86 & \pm & 0,71 & 6,95 & $\pm 0,63$ & 331 & \pm & 124 \\
\hline $\begin{array}{l}\text { Weiße gehörnte } \\
\text { Heidschnucke }\end{array}$ & 754 & 7,18 & \pm & 0,56 & 6,90 & \pm & 0,64 & 7,06 & $\pm 0,47$ & 388 & \pm & 150 \\
\hline $\begin{array}{l}\text { Coburger } \\
\text { Fuchsschaf }\end{array}$ & 710 & 7,01 & \pm & 0,57 & 6,93 & \pm & 0,71 & 7,07 & $\pm 0,63$ & 391 & \pm & 150 \\
\hline
\end{tabular}

\section{3. $\quad$ Ergebnisse}

Die Mittelwerte, Standardabweichungen und die Anzahl der Beobachtungen sind für die drei Merkmale und das Erfassungsalter in Tabelle 1 dargestellt. Die Mittelwerte des Merkmals Exterieur waren bei allen Rassen kleiner als beim Merkmal Bemuskelung. Das durchschnittliche Alter bei der Körung/Zuchtbuchaufnahme lag beim Bentheimer Landschaf mit 331 Tagen am niedrigsten, während es bei der Grauen gehörn- 
ten Heidschnucke mit 468 Tagen am höchsten war. Es wies bei allen Rassen hohe Standardabweichungen auf.

Einen Überblick über die Signifikanz der systematischen Effekte für die einzelnen Schafrassen geben die Tabellen 2 bis 4 . Hier zeigte sich, dass der Herde-Jahr-SaisonEffekt für alle Rassen in allen untersuchten Merkmalen signifikant war.

Tabelle 2

Einfluss systematischer Faktoren auf das Merkmal Bonitierung der Bemuskelung (Influence of systematic effects on score for muscle mass)

\begin{tabular}{lccccc}
\hline Rasse & HJS & MTK & Geschlecht & Geburtstyp & Alter \\
\hline $\begin{array}{l}\text { Weiße hornlose } \\
\text { Heidschnucke }\end{array}$ & $* * *$ & n.s. & n.s. & $* * *$ & n.s. \\
$\begin{array}{l}\text { Graue gehörnte } \\
\text { Heidschnucke }\end{array}$ & $* * *$ & $* * *$ & n.s. & $* * *$ & $* * *$ \\
$\begin{array}{l}\text { Bentheimer Landschaf } \\
\text { Weiße gehörnte }\end{array}$ & $* *$ & n.s. & n.s. & $* * *$ & $* *$ \\
$\begin{array}{l}\text { Heidschnucke } \\
\text { Coburger Fuchsschaf }\end{array}$ & $* * *$ & n.s. & $*$ & n.s. & $* * *$ \\
\hline
\end{tabular}

HJS: Herde-Jahr-Saisonklassen; MTK: Messtagsklassen;

n.s.: $\mathrm{p}>0,05 ; *$ : $\mathrm{p} \leq 0,05 ; * *$ : $\leq 0,01 ; * * *$ : $\leq 0,001$

Bei der Betrachtung des Merkmals Bemuskelung (Tab. 2) fällt auf, dass das Alter mit Ausnahme der Weißen hornlosen Heidschnucke eine signifikante Bedeutung hatte. Auch der Geburtstyp hatte einen hohen Einfluss, außer bei den Weißen gehörnten Heidschnucken und den Coburger Fuchsschafen. Der Faktor Geschlecht zeigte mit Ausnahme der Rasse Weiße gehörnte Heidschnucke keinen Einfluss auf die Bemuskelung. Die Messtagsklasse hatte nur bei den Grauen gehörnten Heidschnucken und den Coburger Fuchsschafen eine signifikante Bedeutung.

Tabelle 3

Einfluss systematischer Faktoren auf das Merkmal Bonitierung des Exterieurs (Influence of systematic effects on score for type)

\begin{tabular}{|c|c|c|c|c|c|}
\hline Rasse & HJS & MTK & Geschlecht & Geburtstyp & Alter \\
\hline $\begin{array}{l}\text { Weiße hornlose } \\
\text { Heidschnucke }\end{array}$ & $* * *$ & n.s. & $* *$ & $* * *$ & n.s. \\
\hline $\begin{array}{l}\text { Graue gehörnte } \\
\text { Heidschnucke }\end{array}$ & $* * *$ & $* * *$ & n.s. & $* * *$ & n.s. \\
\hline Bentheimer Landschaf & $* * *$ & $* *$ & n.s. & $*$ & n.s. \\
\hline $\begin{array}{l}\text { Weiße gehörnte } \\
\text { Heidschnucke }\end{array}$ & $* * *$ & n.s. & n.s. & n.s. & n.s. \\
\hline Coburger Fuchsschaf & $* *$ & n.s. & n.s. & n.s. & $*$ \\
\hline
\end{tabular}

HJS: Herde-Jahr-Saisonklassen; MTK: Messtagsklassen;

n.s.: $\mathrm{p}>0,05 ; *$ : $\leq 0,05 ; * *$ : $\leq 0,01 ; * * *$ : $\leq 0,001$

Beim Merkmal Exterieur (Tab. 3) waren zum Teil andere signifikante systematische Einflüsse festzustellen. So hatte das Alter mit Ausnahme der Coburger Fuchsschafe keinen signifikanten Einfluss. Ähnlich verhielt es sich mit dem Geschlecht, welches nur bei den Weißen hornlosen Heidschnucken einen signifikanten Einfluss aufwies. Die Messtagsklasse war bei den Grauen gehörnten Heidschnucken und bei den Bentheimer Landschafen signifikant. Die hohe Bedeutung des Geburtstyps war auch hier offensichtlich. Eine Ausnahme stellten hier nur die Weißen gehörnten Heidschnucken und die Coburger Fuchsschafe dar. 
Das Merkmal Wolle (Tab. 4) wurde vom Alter nicht beeinflusst. Während Messtagsklasse, Geschlecht und Geburtstyp bei den Weißen hornlosen Heidschnucken einen signifikanten Einfluss aufwiesen, spielten diese drei Faktoren bei den Bentheimer Landschafen und den Coburger Fuchsschafen keine Rolle. Bei den Grauen gehörnten Heidschnucken hingegen hatte von diesen drei Faktoren die Messtagsklasse und bei den Weißen gehörnten Heidschnucken das Geschlecht und der Geburtstyp eine signifikante Bedeutung.

Tabelle 4

Einfluss systematischer Faktoren auf das Merkmal Bonitierung der Wollqualität (Influence of systematic effects on score for wool quality)

\begin{tabular}{|c|c|c|c|c|c|}
\hline Rasse & HJS & MTK & Geschlecht & Geburtstyp & Alter \\
\hline $\begin{array}{l}\text { Weiße hornlose } \\
\text { Heidschnucke }\end{array}$ & $* * *$ & $*$ & $* * *$ & $* * *$ & n.s. \\
\hline $\begin{array}{l}\text { Graue gehörnte } \\
\text { Heidschnucke }\end{array}$ & $* * *$ & $* *$ & n.s. & $* * *$ & n.s. \\
\hline Bentheimer Landschaf & $* * *$ & n.s. & n.s. & n.s. & n.s. \\
\hline $\begin{array}{l}\text { Weiße gehörnte } \\
\text { Heidschnucke }\end{array}$ & $* *$ & n.s. & $* *$ & $*$ & n.s. \\
\hline Coburger Fuchsschaf & $* * *$ & n.s. & n.s. & n.s. & n.s. \\
\hline
\end{tabular}

HJS: Herde-Jahr-Saisonklassen; MTK: Messtagsklassen;

n.s.: $\mathrm{p}>0,05 ; *$ : $\mathrm{p} \leq 0,05 ; * *$ : $\mathrm{p} \leq 0,01 ; * * *$ : $\leq 0,001$

\section{Tabelle 5}

Heritabilitäten (Diagonale), additiv-genetische (oberhalb der Diagonale) und residuale (unterhalb der Diagonale) Korrelationen einschließlich deren Standardfehler der Merkmale Bonitierung der Bemuskelung, des Exterieurs und der Wolle für alle Landschafrassen (Heritabilities (on the diagonal), genetic (above the diagonal) and residual (below the diagonal) correlations, and their standard errors for the traits score for muscle mass, type and wool quality in all land sheep breeds)

\begin{tabular}{cccc}
\hline Merkmal & Bemuskelung & Exterieur & Wolle \\
\hline Bemuskelung & $\mathbf{0 , 1 4}(0,01)$ & $0,76(0,06)$ & $0,45(0,06)$ \\
Exterieur & $0,42(0,01)$ & $\mathbf{0 , 0 6}(0,01)$ & $0,56(0,08)$ \\
Wolle & $0,27(0,01)$ & $0,32(0,01)$ & $\mathbf{0 , 1 0}(0,01)$ \\
\hline
\end{tabular}

In Tabelle 5 werden die Heritabilitäten, die additiv-genetischen Korrelationen und die residualen Korrelationen für die gemeinsame Analyse der Landschafrassen in einem Modell dargestellt. So wurde zwischen den Merkmalen Bemuskelung und Exterieur eine hohe additiv-genetische Korrelation geschätzt. Zwischen den Merkmalen Exterieur und Wolle hingegen lag die additiv-genetische Korrelation bei $r_{g}=0,45$. Zwischen der Bemuskelung und der Wolle bestanden noch niedrigere additiv-genetische Korrelationen. Für das Merkmal Bemuskelung wurde eine mittlere Heritabilität von $\mathrm{h}^{2}$ $=0,14$ geschätzt. Niedrigere Heritabilitäten ergaben sich für die Merkmale Wolle $\left(\mathrm{h}^{2}=\right.$ $0,10)$ und Exterieur $\left(h^{2}=0,06\right)$.

Die additiv-genetische Varianz, die Heritabilität und ihre Standardfehler sind für jede einzelne Rasse in Tabelle 6 dargestellt. Die Heritabilitäten für das Merkmal Bemuskelung lagen zwischen $\mathrm{h}^{2}=0,06$ und $\mathrm{h}^{2}=0,16$, für das Merkmal Exterieur zwischen $\mathrm{h}^{2}=0,04$ und $\mathrm{h}^{2}=0,09$ und für das Merkmal Wolle zwischen $\mathrm{h}^{2}=0,03$ und $\mathrm{h}^{2}=$ 0,14. Für die Weißen gehörnten Heidschnucken konnte für die Merkmale Exterieur und Wolle nur eine sehr geringe Heritabilität geschätzt werden. Eine mögliche Ursache könnte in der zugrundeliegenden Datenstruktur liegen. 
Tabelle 6

Heritabilitäten $\left(\mathrm{h}^{2}\right)$, deren Standardfehler (SE) und additiv-genetische Varianzen der Merkmale Bonitierung der Bemuskelung, des Exterieurs und der Wollqualität bei Landschafrassen sowie Anzahl der Tiere im Pedigree (Pedigree) (Heritabilities $\left(\mathrm{h}^{2}\right)$, their standard errors (SE) and additive genetic variances of the traits score for musle mass, type and wool quality for land sheep breeds, number of animals in pedigree (Pedigree))

\begin{tabular}{|c|c|c|c|c|c|c|c|c|c|c|}
\hline \multirow[t]{2}{*}{ Merkmal } & \multirow[b]{2}{*}{ Pedigree } & \multicolumn{3}{|c|}{ Bemuskelung } & \multicolumn{3}{|c|}{ Exterieur } & \multicolumn{3}{|c|}{ Wolle } \\
\hline & & $\sigma_{\mathrm{a}}^{2}$ & $h^{2}$ & SE & $\sigma_{\mathrm{a}}^{2}$ & $h^{2}$ & SE & $\sigma_{a}^{2}$ & $h^{2}$ & SE \\
\hline $\begin{array}{l}\text { Weiße hornlose } \\
\text { Heidschnucke }\end{array}$ & 7511 & 0,06 & 0,12 & 0,02 & 0,03 & 0,06 & 0,02 & 0,02 & 0,03 & 0,02 \\
\hline $\begin{array}{l}\text { Graue gehörnte } \\
\text { Heidschnucke }\end{array}$ & 6714 & 0,07 & 0,13 & 0,03 & 0,03 & 0,06 & 0,02 & 0,02 & 0,05 & 0,03 \\
\hline Bentheimer Landschaf & 5251 & 0,04 & 0,13 & 0,02 & 0,04 & 0,09 & 0,02 & 0,05 & 0,14 & 0,03 \\
\hline $\begin{array}{l}\text { Weiße gehörnte } \\
\text { Heidschnucke }\end{array}$ & 1338 & 0,02 & 0,06 & 0,04 & 0,00 & 0,00 & 0,01 & 0,00 & 0,01 & 0,02 \\
\hline Coburger Fuchsschaf & 1692 & 0,04 & 0,16 & 0,11 & 0,02 & 0,04 & 0,06 & 0,05 & 0,13 & 0,05 \\
\hline
\end{tabular}

\section{4.}

\section{Diskussion}

Die hier untersuchten Rassen wiesen für die drei untersuchten Merkmale Mittelwerte über 7 bei Standardabweichungen von 0,5 bis 0,8 auf. Daran ist zu erkennen, dass die Punkteskala von 1 bis 9, insbesondere im unteren Bereich, nicht ausgenutzt wird. Dies hängt damit zusammen, dass bei der Körung und Herdbuchaufnahme nur solche Tiere bewertet werden, mit denen auch weiter gezüchtet werden soll. Dieser Sachverhalt wurde für die Bonitierung der Bemuskelung bereits von STIER et al. (1988) bemängelt. Sie wiesen darauf hin, dass die subjektive Typbewertung bei vollständiger Ausnutzung der Punkteskala eine ausreichende genetische Varianz aufweisen könnte. Auch SAVAS et al. (2001) wiesen darauf hin, dass die Punkteskala im Zuchtverband Schleswig-Holsteinischer Schafzüchter e.V. in der subjektiven Bewertung der Bemuskelung und der äußeren Erscheinung nicht vollständig ausgeschöpft wurde. Allerdings beziehen sich beide Untersuchungen auf Fleischschafrassen. Dass die Mittelwerte des Merkmals Exterieur immer unter den Mittelwerten des Merkmals Bemuskelung liegen, wurde bereits von LAUE (1984) als auch von SAVAS et al. (2001) für Fleischschafrassen festgestellt. Die Landschafrassen werden in der Regel als Jährlinge ins Herdbuch aufgenommen. Dies ist am deutlichsten bei den Grauen gehörnten Heidschnucken zu erkennen, da sie ein durchschnittliches Alter von 469 Tagen besitzen. Die hohen Standardabweichungen sowie die etwas niedrigeren Durchschnittswerte für die anderen Landschafrassen weisen darauf hin, dass die Art der Aufnahme von Zuchttieren von den Verbänden unterschiedlich durchgeführt wird. So wird neben der Aufnahme der Jährlinge auch in einigen Verbänden eine Aufnahme im Lammalter vorgenommen. Letztendlich führt dieses unterschiedliche Vorgehen zu einer bimodalen Verteilung hinsichtlich des Alters, was die Auswertung erschwert.

Der Herde-Jahr-Saison-Effekt weist in allen Merkmalen einen hohen, signifikanten Einfluss auf. Zum einen spielen wechselnde Witterungsverhältnisse, die beispielsweise die Futterbedingungen im Herbst und Frühjahr beeinflussen, eine große Rolle, zum anderen aber auch Betriebseffekte, wie Standort, Fütterungsintensität, Aufzuchtverfahren, Herdengröße und Management des Betriebsleiters.

In allen drei Merkmalen zeigte sich bei den Rassen, bei denen der Geburtstyp einen signifikanten Einfluss aufwies, dass Einlingslämmer grundsätzlich höhere Leistungen als Zwillings- und/oder Drillingslämmer hatten. Auch zeigten männliche Tiere höhere 
Leistungen als weibliche Tiere mit Ausnahme der Weißen gehörnten Heidschnucke im Merkmal Exterieur. Hier waren die weiblichen Tiere signifikant überlegen.

Die in dieser Studie beschriebenen hohen genetischen Korrelationen für die subjektive Bewertung der Bemuskelung und des Exterieurs stimmen mit den Analysen von SAVAS et al. (2001) bei Fleischschafrassen überein. Gegenüber den Fleischschafen und Milchschafen (DE VRIES et al., 2003) zeigten sich hier höhere genetische Korrelationen zwischen den Merkmalen Bemuskelung und Wolle sowie Exterieur und Wolle.

Bisher wurden keine Heritabilitäten für die subjektive Bewertung der Bemuskelung, des Exterieurs und der Wolle bei Landschafrassen geschätzt. In der Literatur wurden nur für Fleischschaf- und Milchschafrassen für diese Merkmale Heritabilitäten geschätzt. So erhielt LAUE (1984) bei den Rassen Weißköpfiges Fleischschaf, Schwarzköpfiges Fleischschaf und Texel einen Wert von $\mathrm{h}^{2}=0,25$ für die Erblichkeit der subjektiven Bewertung der Bemuskelung. Niedrige Heritabilitäten für die Bemuskelung wurden von STIER et al. (1988) $\mathrm{mit}^{2}=0,15$ für das Schwarzköpfige Fleischschaf und $h^{2}=0,09$ für das Merinofleischschaf angegeben. MÜLLER (1995) schätzte dagegen höhere Werte für die Feldprüfung der Bemuskelung beim Merinofleischschaf. Von SAVAS et al. (2001) wurden für vier Fleischschafrassen Heritabilitäten für die Bemuskelung und die äußere Erscheinung geschätzt. Diese Werte lagen etwas unter den von MÜLLER (1995) geschätzten Werten. Für niedersächsische Fleischschafrassen und Milchschafrassen wurden ähnliche Werte geschätzt (DE VRIES et al., 2003). Die in der vorliegenden Arbeit für Landschafe geschätzten Werte zeigen im Vergleich mit den Schätzwerten aus der Literatur für Fleisch- und Milchschafrassen im mittleren Bereich liegende Werte.

Grundsätzlich ist zu überlegen, ob eine Selektion auf diese Merkmale wie bei den Fleisch- und Milchschafrassen erfolgen sollte. Die Besonderheit der Landschafe ist ihre hervorragende Eignung für die Landschaftspflege. Zur Beurteilung dieser Eigenschaften sollten Merkmale wie Körper- und Klauenmaße eingeführt werden, um diese bei der Körung und Herdbuchaufnahme mit zu erheben. Neben der subjektiven Bewertung ermöglichen diese Merkmale eine objektiven Beurteilung der Nutzung zur Landschaftspflege. Auch wenn die oben analysierten Merkmale nicht unmittelbar die Eignung zur Landschaftspflege wiedergeben, können sie doch das Aussehen des Tieres beschreiben und damit verdeutlichen, wie sich das Tier unter extremen Bedingungen entwickelt hat. Aus diesem Grunde sollte bei der Körung und Herdbuchaufnahme immer mitbeachtet werden, unter welchen Bedingungen das Tier gehalten wird.

5. Schlussfolgerungen für die Praxis

Die in dieser Studie analysierten Merkmale sind für die Selektion der Landschafe in der Zucht geeignet. Sie beeinflussen sich gegenseitig positiv und die Heritabilitäten sind ausreichend hoch. Außerdem kann das Ziel der Landschaftspflege unter Berücksichtigung der Haltung mit in diese Merkmale einfließen. Die Zucht auf Eignung zur Landschaftspflege kann allerdings durch objektive Parameter ergänzt und verbessert werden.

\section{Danksagung}

Dem Niedersächsischen Ministerium für den ländlichen Raum, Ernährung, Landwirtschaft und Forsten sowie der Tierseuchenkasse Niedersachsens gilt besonderer Dank 
für die finanzielle Unterstützung des Projektes. Den Schafzuchtverbänden Landesschafzuchtverband Niedersachsen e.V., Stader Schafzuchtverband e.V., Landes-Schafzuchtverband Weser-Ems e.V., Verband Lüneburger Heidschnuckenzüchter e.V. und der Vereinigung Westfälischer Herdbuchschafzüchter e.V. sei herzlich für die zur Verfügung gestellten Daten gedankt.

\section{Literatur}

DE VRIES, F.; BORCHERS, N.; DRÖGEMÜLLER, C.; HAMANN, H.; REINECKE, S.; LÜPPING, W.; DISTL, O.:

Analyse der Assoziation zwischen den Prionproteingenotypen und Leistungsmerkmalen bei Fleischschafrassen. Züchtungskunde 75 (2003), 249-258

GROENEVELD, E.:

VCE4 User's Guide and Reference Manual Version 1.1. Institute for Animal Science and Animal Husbandry, Federal Agricultural Research Centre (Bundesforschungsanstalt für Landwirtschaft, FAL), LAUE, H.-J.: Mariensee / Neustadt (1998), Germany

Untersuchungen zur Verbesserung der Fleischleistungsprüfung und Zuchtwertschätzung beim Schaf. Schriftenreihe des Institutes für Tierzucht und Tierhaltung der Christian-Albrechts-Universität zu Kiel, Heft 15, 1984

MÜLLER, U.:

Planungsrechnungen zur Optimierung von Zuchtsystemen für die Verbesserung der Mastleistung und Schlachtkörperqualität der Rasse Merinofleischschaf unter Verwendung des Ultraschallmessverfahrens am lebenden Tier. Dissertation agr. (1995), Universität Leipzig

SAS:

Cary, NC: SAS Institute Inc., Version 8.2, 2003

STIER, C.-H.; VON KORN, ST.; PETERS, K.J.:

Fleischleistungsprüfungen in der Schafzucht. 1. Mitteilung: Der Einfluss systematischer Faktoren im Feld. Züchtungskunde 60 (1988), 62-71

STIER, C.-H.; VON KORN, ST.; PETERS, K.J.:

Fleischleistungsprüfungen in der Schafzucht. 3. Mitteilung: Genetische Fundierung der im Feld und auf Station erfassten Mastleistungskriterien sowie Ansätze zur Optimierung der niedersächsischen Fleischleistungsprüfungen beim Schaf. Züchtungskunde 60 (1988), 123-134

SAVAS, T.; RÖHE, R.; KALM, E.:

Genetisch-statistische Analyse der Mastleistungs- und Schlachtkörperwertmerkmale von Fleischschafrassen aus der Feldprüfung von Schleswig-Holstein. Züchtungskunde 73 (2001), 62-73

Eingegangen: 05.11.2003

Akzeptiert: 23.06.2004

Anschrift der Verfasser FRERICH DE VRIES, Dr. HENNING HAMANN, Prof. Dr. OTTMAR DISTL

Institut für Tierzucht und Vererbungsforschung

Bünteweg 17p

D-30559 Hannover

E-Mail: frerich.de.vries@tiho-hannover.de, ottmar.distl@tiho-hannover.de 